\title{
Arginine-Modified Polymers Facilitate Poly (Lactide-Co-Glycolide)-Based Nanoparticle Gene Delivery to Primary Human Astrocytes
}

This article was published in the following Dove Press journal: International Journal of Nanomedicine

\author{
Jessica Proulx (D) \\ Chaitanya Joshi $\mathbb{1}^{\prime}$ \\ Sivakumar Vijayaraghavalu (iD) ${ }^{2}$ \\ Manju Saraswathy ${ }^{2}$ \\ Vinod Labhasetwar (iD ${ }^{2}$ \\ Anuja Ghorpade' \\ Kathleen Borgmann (1) 1,3 \\ 'Department of Microbiology, \\ Immunology, and Genetics University of \\ North Texas Health Science Center, Fort \\ Worth, TX, USA; ${ }^{2}$ Department of \\ Biomedical Engineering, Lerner Research \\ Institute, Cleveland Clinic, Cleveland, \\ $\mathrm{OH}$, USA; ${ }^{3}$ Department of Pharmacology \\ and Neuroscience, University of North \\ Texas Health Science Center, Fort \\ Worth, TX 76107, USA
}

Correspondence: Kathleen Borgmann Department of Pharmacology and Neuroscience, University of North Texas Health Science Center, 3500 Camp Bowie Blvd, Fort Worth, TX 76107, USA

Tel +I 817 735-0339

Fax $+1817735-2610$

Email Kathleen.Borgmann@unthsc.edu
Purpose: Astrocyte dysfunction is a hallmark of central nervous system injury or infection. As a primary contributor to neurodegeneration, astrocytes are an ideal therapeutic target to combat neurodegenerative conditions. Gene therapy has arisen as an innovative technique that provides excellent prospect for disease intervention. Poly (lactide-co-glycolide) (PLGA) and polyethylenimine (PEI) are polymeric nanoparticles commonly used in gene delivery, each manifesting their own set of advantages and disadvantages. As a clinically approved polymer by the Federal Drug Administration, well characterized for its biodegradability and biocompatibility, PLGA-based nanoparticles (PLGA-NPs) are appealing for translational gene delivery systems. However, our investigations revealed PLGA-NPs were ineffective at facilitating exogenous gene expression in primary human astrocytes, despite their success in other cell lines. Furthermore, PEI polymers illustrate high delivery efficiency but induce cytotoxicity. The purpose of this study is to develop viable and biocompatible NPsystem for astrocyte-targeted gene therapy.

Materials and Methods: Successful gene expression by PLGA-NPs alone or in combination with arginine-modified PEI polymers $\left(\mathrm{A}_{n} \mathrm{P}_{n}\right)$ was assessed by a luciferase reporter gene encapsulated in PLGA-NPs. Cytoplasmic release and nuclear localization of DNA were investigated using fluorescent confocal imaging with YOYO-labeled plasmid DNA (pDNA). NP-mediated cytotoxicity was assessed via lactate dehydrogenase in primary human astrocytes and neurons.

Results: Confocal imaging of YOYO-labeled pDNA confirmed PLGA-NPs delivered pDNA to the cytoplasm in a dose and time-dependent manner. However, co-staining revealed pDNA delivered by PLGA-NPs did not localize to the nucleus. The addition of $A_{n} P_{n}$ significantly improved nuclear localization of pDNA and successfully achieved gene expression in primary human astrocytes. Moreover, these formulations were biocompatible with both astrocytes and neurons.

Conclusion: By co-transfecting two polymeric NPs, we developed an improved system for gene delivery and expression in primary human astrocytes. These findings provide a basis for a biocompatible and clinically translatable method to regulate astrocyte function during neurodegenerative diseases and disorders.

Keywords: co-polymer, transfection, gene therapy, astrocyte targeting, nuclear entry

\section{Plain Language Summary}

Astrocytes are one of the most abundant cells in the brain and provide critical support for neuronal function and survival. During conditions of neurodegeneration, astrocytes can switch from being neuroprotective to become neurotoxic. This role change is a key 
characteristic in the development and severity of central nervous system (CNS) pathologies. Gene therapy intervenes at a DNA level to regulate astrocytes by restoring neuroprotective functions and/or reversing neurotoxicity. Successful gene therapy methods must be safe for neuronal cells and effective at delivering a gene of interest to the target cell. Here, we combined two polymeric nanoparticle (NP) systems with opposing advantages and disadvantages to improve gene delivery, toxicity and expression in astrocytes. Enhanced gene expression was achieved by increasing intracellular and nuclear delivery, without inducing toxicity to astrocytes or neurons. This study addresses a critical challenge of gene delivery to primary human astrocytes and demonstrates how a co-polymer NP formulation can improve gene therapy to these neural cells. In addition, these findings describe an enhanced method that can be used to better study and/or treat astrocyte-mediated neurotoxicity during CNS pathologies.

\section{Introduction}

Astrocytes are the most numerous glial cells in the brain and are integral in maintaining blood-brain barrier (BBB) integrity, central nervous system (CNS) homeostasis, and synaptic transmission. Following CNS injury, astrocytes often undergo a shift in their gene expression exhibiting an "activated" or "inflammatory" phenotype contributing to and exacerbating CNS pathologies. ${ }^{1}$ Gene therapy allows for molecular intervention to regulate astrocyte function to either restore a neuroprotective or reverse a neurotoxic phenotype. ${ }^{2}$ Polymeric NPs are advantageous for gene delivery due to their low immunogenicity, high biocompatibility, versatility, and protection against peripheral and intracellular degradation of the encapsulated nucleic acids. $^{2-5}$

Poly (lactide-co-glycolide) polymers (PLGA) and polyethylenimine (PEI) are two extensively investigated polymers for drug/gene delivery applications. Well regarded for their biocompatibility and sustained release, PLGA-NPs are biodegradable and easily metabolized by the citric acid cycle. ${ }^{3,6}$ Furthermore, PLGA polymers have been approved by the Federal Drug Administration (FDA) for therapeutic applications since 1969 making them preferred NP candidates for clinically translatable gene delivery. ${ }^{6-8}$ However, our studies have revealed PLGAbased NPs encapsulating plasmid DNA (pDNA) as an inefficient gene delivery system to human astrocytes, although these have been shown to transfect other cell lines. $^{9-11}$ On the other hand, PEI-based polymers are often recognized as the most efficient cationic polymer to date, establishing efficient gene delivery and expression by enhancing intracellular trafficking. ${ }^{5,12,13}$ However, clinical trials with PEI failed to progress due to a high induction of cytotoxicity given its high positive surface charge. ${ }^{8}$ More recently, attempts were made to reduce PEI-associated toxicity by surface modifications. In this context, our collaborative work reported novel arginine-modified, polyethylene glycol-stabilized PEI polyplex as a less cytotoxic alternative with the ability to further enhance cellular uptake of pDNA and small interfering RNA. ${ }^{14-16}$

Finding an effective and biocompatible delivery system to regulate astrocyte function is of great importance to restore CNS homeostasis during neurological diseases and disorders. The current study sought a NP gene delivery method for astrocytes using FDA-approved PLGA polymers. Our investigations identified an unanticipated limitation of PLGA-NPs in their ability to effectively deliver and facilitate exogenous gene expression in astrocytes. By combining transfection with arginine-modified PEI-based polymers $\left(\mathrm{A}_{\mathrm{n}} \mathrm{P}_{\mathrm{n}}\right)$, we resolved PLGA-NP-mediated gene delivery to astrocytes while ensuring biocompatibility in both astrocytes and neurons. These findings propose an improved method for polymeric-NP gene therapy to astrocytes which is able to enhance NP uptake, pDNA nuclear localization, and gene expression efficacy.

\section{Materials and Methods}

\section{Tissue Culture and Isolation}

Human Embryonic Kidney 293 T cells (HEKs) were obtained from the American Type Culture Collection (Manassas, VA, USA) and frozen at P4 according to their cryopreservation procedures. HEKs were subcultured and plated at 100,000 cells/well for 24 hours prior to transfection. Primary human astrocytes and neurons were harvested from brain tissues provided by the Birth Defects Research Laboratory, University of Washington as previously described. ${ }^{15,17}$ Tissues were collected from first and early second trimester specimens with written informed consent from the donors, in full compliance with federal laws and the ethical guidelines of the National Institutes of Health and the Declaration of Helsinki. These studies were approved by the University of Washington IRB and the North Texas Regional IRB at the University of North Texas Health Science Center. Astrocytes were plated 24 hours before transfection at 150,000 cells/well in 48-well plates for cytotoxicity and gene expression assays, and at 100,000 cells/well in either 48-well plates or 24-well glass-bottom plates for 
Table I Cell/Donor Information

\begin{tabular}{|c|c|c|c|c|c|}
\hline Cell Culture Type & Donor Number & Gestational Age & Sex & Passage/Week in Culture & Expt./Fig. \# \\
\hline Human Embryonic Kidney 293 & $\mathrm{~N} / \mathrm{A}$ & $\mathrm{N} / \mathrm{A}$ & $N / A$ & P42 & Figure IA \\
\hline Primary Human Astrocytes & $\begin{array}{l}\# 1 \\
\# 2 \\
\# 3 \\
\# 4 \\
\# 5 \\
\# 6\end{array}$ & $\begin{array}{l}105 d \\
117 d \\
96 d \\
122 d \\
105 d \\
125 d\end{array}$ & $\begin{array}{l}\text { Male } \\
\text { Male } \\
\text { Female } \\
\text { Female } \\
\text { Female } \\
\text { Female }\end{array}$ & $\begin{array}{l}\text { P5 } \\
\text { P5 } \\
\text { P3 } \\
\text { P2 } \\
\text { P4 } \\
\text { P4 }\end{array}$ & $\begin{array}{l}\text { Figures } I A \text { and } 3 A-C \\
\text { Figure } I B \\
\text { Figure } 2 A \text { and } B \\
\text { Figure } 2 A \text { and } B \\
\text { Figure } 2 A \text { and } B \\
\text { Figure } 2 A \text { and } B\end{array}$ \\
\hline Primary Human Neurons & $\begin{array}{l}\# 1 \\
\# 2 \\
\# 3\end{array}$ & $\begin{array}{l}57 d \\
76 d \\
77 d\end{array}$ & $\begin{array}{l}\text { Female } \\
\text { Male } \\
\text { Female }\end{array}$ & $\begin{array}{l}\text { W3 } \\
\text { W4 } \\
\text { W3 }\end{array}$ & $\begin{array}{l}\text { Figure } 2 \mathrm{C} \\
\text { Figure } 2 \mathrm{C} \\
\text { Figure } 2 \mathrm{C}\end{array}$ \\
\hline
\end{tabular}

fluorescent and confocal imaging, respectively. Neurons were plated on poly-d-lysine-coated plates for two to four weeks before transfection at 100,000 cells/well in 48well plates. Additional cell culture/donor information is listed in Table 1.

\section{NP Gene Delivery}

Lyophilized PLGA-based NPs encapsulating luciferase pDNA (PLGA/pDNA-NPs) and $\mathrm{A}_{5} \mathrm{P}_{10}$ polymers were received from the lab of Dr. Vinod Labhasetwar in the Department of Biomedical Engineering, Lerner Research Institute, Cleveland Clinic, Cleveland, $\mathrm{OH}$, USA and stored at $-20^{\circ} \mathrm{C}$. Synthesis and characterization of PLGA/pDNA$\mathrm{NPs}^{11}$ and $\mathrm{A}_{\mathrm{n}} \mathrm{P}_{\mathrm{n}}$ polymers ${ }^{15}$ were described previously. For co-polymer gene delivery, PLGA/pDNA-NPs were first resuspended in PBS to $2 \mu \mathrm{g} / \mu \mathrm{L}$ with water-bath sonication for five minutes. The $\mathrm{A}_{5} \mathrm{P}_{10}$ polymers were separately resuspended to $1 \mu \mathrm{g} / \mu \mathrm{L}$ in PBS. Co-polymer transfection solutions were prepared by diluting each stock into fresh media to the desired concentrations, pipetting to mix and then fully exchanging culture media with the prepared solutions. We did not manipulate or induce any physicochemical process that would alter the structure of polymers. Each transfection condition was carried out in triplicate wells with PLGA/ pDNA-NPs (60 or $120 \mu \mathrm{g} /$ well), $\mathrm{A}_{5} \mathrm{P}_{10}$ ( 1 or $2 \mu \mathrm{g} /$ well), and luciferase pDNA ( $1 \mu \mathrm{g} /$ well) when indicated, for six hours to six days before analysis. Luciferase was used as a reporter gene to assess gene expression. For NP characterization (Table 2), PLGA/pDNA-NPs were resuspended and measured at $500 \mu \mathrm{g} / \mathrm{mL}$ using Zetasizer Nano Series (Malvern Panalytical Ltd., United Kingdom). $\mathrm{A}_{5} \mathrm{P}_{10}(1 \mu \mathrm{g} /$ $\mu \mathrm{L}$ ) was added at the indicated ratios in molecular grade water for characterization of co-polymer conditions
(PLGA/pDNA $+\mathrm{A}_{5} \mathrm{P}_{10}$ ). The highest concentration of $\mathrm{A}_{5} \mathrm{P}_{10}$ used was $16.66 \mu \mathrm{g} / \mathrm{mL}$ (for $30: 1$ ), which was also used for $\mathrm{A}_{5} \mathrm{P}_{10}$ alone measurements.

\section{Gene Expression}

Based on the expression profiles of PLGA/pDNA-NPs ${ }^{6-8,11}$ and $\mathrm{A}_{\mathrm{n}} \mathrm{P}_{\mathrm{n}} / \mathrm{pDNA}-\mathrm{NPs},{ }^{15}$ gene expression was measured via luciferase assay 48 hours post-transfection. Cells were lysed in 1x passive lysis buffer for 20 minutes then measured in duplicated determinations for rapid detection of firefly luciferase activity according to the manufacturer's instructions (cat. \#E1500, Promega, Madison, WI, USA).

\section{Cytotoxicity}

Cytotoxicity was measured via lactate dehydrogenase (LDH) assay 48 hours post-transfection. Duplicate collections of $50 \mu \mathrm{L}$ of supernatant per well were incubated 1:1 with LDH reaction buffer from Cytotoxicity Detection Kit

Table 2 NP Characterization

\begin{tabular}{|l|l|l|l|}
\hline Construct & Size (nm) & $\begin{array}{l}\text { Polydispersity } \\
\text { Index }\end{array}$ & $\begin{array}{l}\text { Zeta } \\
\text { Potential } \\
(\mathrm{mV})\end{array}$ \\
\hline $\mathrm{A}_{5} \mathrm{P}_{10}$ alone & $155.8 \pm 6.2$ & $0.28 \pm 0.05$ & $7.72 \pm 2.93$ \\
\hline $\begin{array}{l}\mathrm{PLGA} / \mathrm{pDNA}- \\
\mathrm{NPs}\end{array}$ & $331.6 \pm 20.5$ & $0.39 \pm 0.04$ & $-2.59 \pm 0.26$ \\
\hline $\begin{array}{l}\mathrm{PLGA} / \mathrm{pDNA}+ \\
\mathrm{A}_{5} \mathrm{P}_{10}(\mathrm{I} 20: 1)\end{array}$ & $361.6 \pm 22.6$ & $0.44 \pm 0.03$ & $-1.67 \pm 0.12$ \\
\hline $\begin{array}{l}\mathrm{PLGA} / \mathrm{PDNA}+ \\
\mathrm{A}_{5} \mathrm{P}_{10}(60: 1)\end{array}$ & $342.4 \pm 6.3$ & $0.38 \pm 0.02$ & $-1.09 \pm 0.06$ \\
\hline $\begin{array}{l}\mathrm{PLGA} / \mathrm{PDNA}+ \\
\mathrm{A}_{5} \mathrm{P}_{10}(30: 1)\end{array}$ & $344.5 \pm 7.0$ & $0.42 \pm 0.03$ & $-0.69 \pm 0.40$ \\
\hline
\end{tabular}


(\#11644793001, Sigma Aldrich, St. Louis, MO, USA) for 25 minutes (astrocytes) or 45-60 minutes (neurons) in the dark. Absorbance was read at $490 \mathrm{~nm}$ with background correction at $650 \mathrm{~nm}$.

\section{Live Cell Imaging}

Cells were imaged in 1x ProLong Live Antifade Reagent (cat. \# P36975, ThermoFisher, Waltham, MA, USA) in Hanks Balanced Salt Solution at 6, 24, 48, or 96 hours post-transfection with luciferase pDNA labeled with YOYO fluorescent dye. ${ }^{14}$ Fluorescent images were acquired with 40X objective with Nikon Eclipse Ti-4 using the NIS-Elements BR 3.2 software (Nikon, Melville, NY, USA). Cells analyzed for colocalization were incubated for 10-30 minutes with Hoechst nuclear dye (cat. \# H1399, ThermoFisher) prior to confocal imaging. Z-stack images were taken at 40x magnification with excitation and emission at 491/509 $\mathrm{nm}$ for YOYO and $350 / 461 \mathrm{~nm}$ for Hoechst. Colocalization coefficients were obtained via LSM 510 Meta Zeiss colocalization tool after adjusting to maximum fluorescent intensity projection (Zeiss, Germany).

\section{Statistical Significance}

Due to high genetic variation among primary brain tissue cultures, similar trends in three or more donors were considered biologically significant for statistical evaluation. One-way ANOVA was used to analyze LDH and colocalization coefficients, and two-way ANOVA was used for luciferase activity. Tukey's multiple comparisons post hoc test was used to determine significance between transfection groups. $\mathrm{P}$ values equal to or less than 0.05 were considered statistically significant.

\section{Results}

\section{PLGA-Mediated Gene Delivery to Primary Human Astrocytes}

Our previous collaborative work reported safe and effective protein delivery to primary neural cells including astrocytes and neurons using PLGA-NPs. ${ }^{18,19}$ Similarly, preliminary studies also indicated that PLGA-NP can effectively deliver and express reporter pDNA in HEKs and immortalized human astrocytes (data not shown). Therefore, the potential of PLGA/pDNA-NPs for gene delivery to human astrocytes was investigated using pDNA encoding a luciferase reporter gene. HEKs were used as a positive control for PLGA/ pDNA-NP-mediated gene delivery. Astrocytes and HEKs were treated with PLGA/pDNA-NPs $(120 \mu \mathrm{g})$ for 2,3 , and 6 days followed by a luciferase expression assay. Luciferase expression increased in a time-dependent manner in HEKs but failed to express luciferase in astrocytes at all tested time points (Figure 1A). To determine if PLGA/pDNA-NPs were able to cross the plasma membrane in astrocytes, YOYO-labeled luciferase pDNA (YOYO-pDNA) encapsulated in PLGA-NPs were treated at 60 or $120 \mu \mathrm{g}$ then imaged at 6, 24, 48, and 96 hours using live cell fluorescent microscopy. YOYO fluorescence increased in a timedependent manner with no obvious differences between concentrations (Figure 1B). These findings confirmed PLGA-NPs with encapsulated pDNA were internalized by astrocytes, suggesting that a possible intracellular mechanism was preventing successful gene expression.

\section{Gene Delivery and Biocompatibility of PLGA/pDNA+ $A_{5} P_{10}$ Co-Transfection}

We recently identified $\mathrm{A}_{n} \mathrm{P}_{n}$ as an effective gene delivery system to primary human astrocytes and neurons. Among the six $A_{n} P_{n}$ constructs and four concentrations tested, $\mathrm{A}_{5} \mathrm{P}_{10}$ polymers at $1-2 \mu \mathrm{g} / \mathrm{well}$ were chosen for the current investigations based on their overall transfection efficiency and cytocompatibility. ${ }^{15}$ Optimizing a gene delivery system employing PLGA/pDNA-NPs targeting astrocytes could promote clinical translation as PLGA is an FDAapproved polymer and provides additional therapeutic benefits such as biodegradability and sustainability. ${ }^{6-8,11} \mathrm{We}$ hypothesized that by combining $\mathrm{A}_{5} \mathrm{P}_{10}$ polymers with PLGA/pDNA-NP transfection, we could improve PLGA/ pDNA-NP-mediated intracellular trafficking of pDNA and subsequent gene expression in astrocytes. Characterization of PLGA/pDNA-NPs and $\mathrm{A}_{5} \mathrm{P}_{10}$, alone and in combination, is illustrated in Table 2. Slight increases in NP size and modest decreases in Zeta Potential indicate minimal changes to PLGA/pDNA-NP construct when combined with $\mathrm{A}_{5} \mathrm{P}_{10}$. Astrocytes were treated with $\mathrm{PLGA} / \mathrm{pDNA}$ NPs $(60$ or $120 \mu \mathrm{g})$ harboring luciferase reporter gene alone or in combination with $\mathrm{A}_{5} \mathrm{P}_{10}$ polymer $(1$ or $2 \mu \mathrm{g})$ for 48 hours. Co-transfection of PLGA/pDNA-NPs with $\mathrm{A}_{5} \mathrm{P}_{10}$ significantly enhanced luciferase activity compared to PLGA/pDNA-NPs alone (Figure $2 \mathrm{~A}, \mathrm{p}<0.001$ ). This phenomenon increased with $\mathrm{A}_{5} \mathrm{P}_{10}$ concentration, highlighting $\mathrm{A}_{5} \mathrm{P}_{10}$ as a key factor facilitating gene expression in astrocytes during co-transfection. This same trend was evident with two additional arginine-modified PEI constructs $\left(\mathrm{A}_{1} \mathrm{P}_{10}\right.$ and $\left.\mathrm{A}_{5} \mathrm{P}_{50}\right)$ (data not shown). 


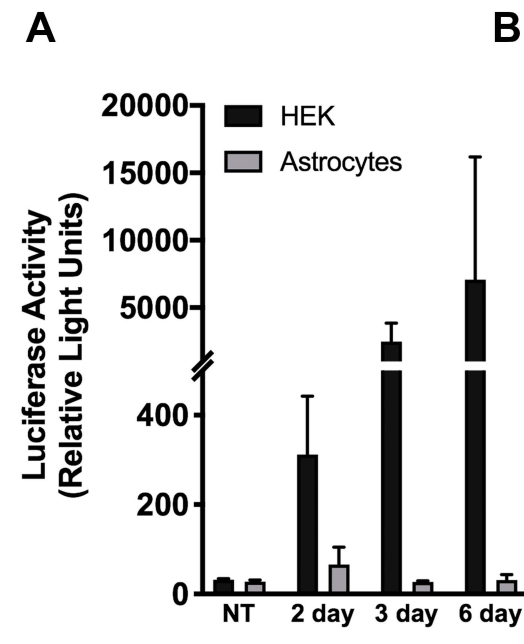

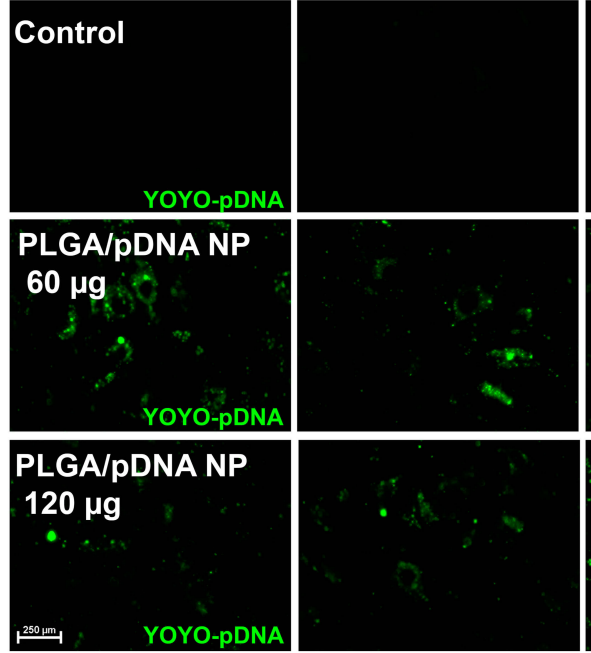

$6 \mathrm{~h}$

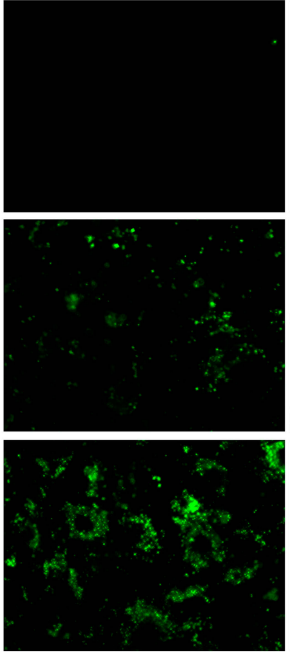

$48 \mathrm{~h}$

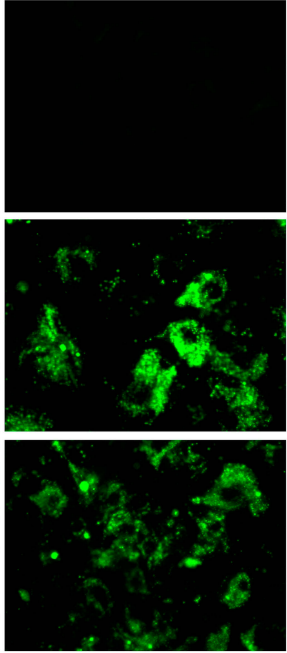

$96 \mathrm{~h}$

Figure I PLGA/PDNA-NPs were taken up by astrocytes but failed to facilitate exogenous gene expression. Luciferase reporter pDNA encapsulated in PLGA-NPs was used to assess PLGA-mediated gene delivery (A). HEKs or primary human astrocytes were treated with $120 \mu g$ PLGA/pDNA-NPs for 2, 3, and 6 days. Luciferase reporter assay was used to verify exogenous gene expression. Data are graphed as mean \pm SD from a representative experiment with duplicated measurements from triplicate wells. Two additional experiments illustrated the same trend. (B) Primary human astrocytes were treated with YOYO-pDNA encapsulated in PLGA-NPs (60 or I20 $\mu$ g) and imaged at 6, 24, 48 and 96 hours using fluorescent microscopy to gauge PLGA-NP-mediated pDNA delivery to the cytoplasm.

Biocompatibility of the integrated delivery system was measured in both primary human astrocytes and neurons by measuring LDH activity as an indicator of cytotoxicity. There was a marginal increase in astrocyte toxicity with PLGA/pDNA-NPs $(120 \mu \mathrm{g})$ transfection alone, while cotransfection paradigms were more comparable to control (Figure 2B). Cytotoxicity in primary neurons was significantly induced by $\mathrm{A}_{5} \mathrm{P}_{10}(2 \mu \mathrm{g})$ alone but was abrogated when co-treated with PLGA/pDNA-NPs (Figure 2C, $\mathrm{p}<0.01)$. Altogether, these data supportthe combination of PLGA/pDNA-NPs with $\mathrm{A}_{5} \mathrm{P}_{10}$ as an efficient and biocompatible method for gene delivery to primary human astrocytes.

\section{Intracellular Delivery of YOYO-pDNA by $\mathrm{PLGA} / \mathrm{PDNA}+\mathrm{A}_{5} \mathrm{P}_{10}$ Co-Transfection}

To ascertain how $\mathrm{A}_{5} \mathrm{P}_{10}$ enhanced PLGA/pDNA-NP-mediated gene delivery and expression, the nuclei of live astrocytes were labeled with Hoechst at 24 hours post-transfection with YOYO-pDNA. Z-stack images were then acquired using confocal microscopy followed by colocalization analysis (Figure $3 \mathrm{~A}$ and B). PLGA/pDNA-NPs encapsulating YOYO-pDNA $(60$ or $120 \mu \mathrm{g}$ ) were transfected alone or in combination with $\mathrm{A}_{5} \mathrm{P}_{10}$ polymers $(2 \mu \mathrm{g})$. YOYO-pDNA delivered by $\mathrm{A}_{5} \mathrm{P}_{10}$ were used as a positive control. Compared to PLGA/pDNANP delivery alone, co-transfection with $\mathrm{A}_{5} \mathrm{P}_{10}$ resulted in
A

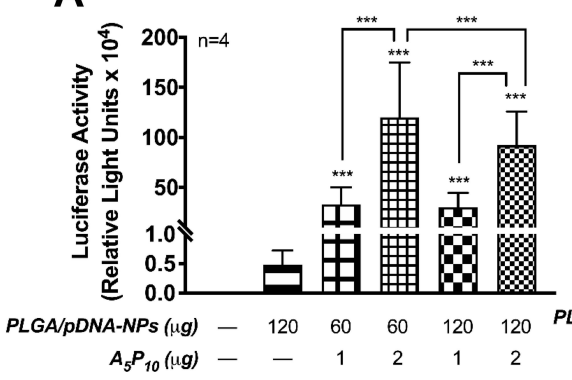

B

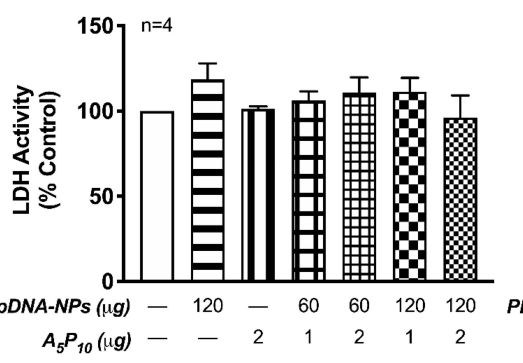

C

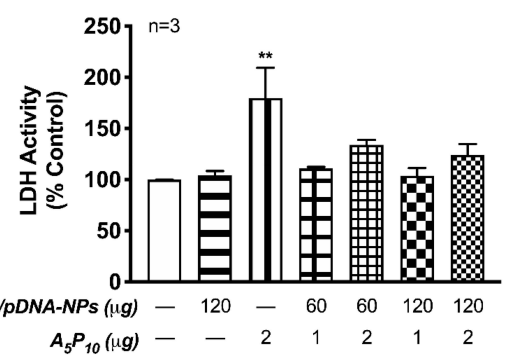

Figure 2 Co-transfection of PLGA/PDNA-NPs with $A_{5} P_{10}$ established an efficient and biocompatible mechanism for astrocyte gene therapy. Primary human astrocytes $(\mathbf{A}$ and $\mathbf{B})$ or neurons $(\mathbf{C})$ were treated with luciferase pDNA encapsulated in PLGA-NPs $(60$ or $120 \mu g)$ alone or with $A_{5} P_{10}$ polymer $(I$ or $2 \mu g)$ for 48 hours. $(\mathbf{A})$ Luciferase activity was quantified using luciferase expression assay. The $x$-axis was broken to indicate the scale in luciferase activity, ie, bottom $\left(0-I\right.$ RLU $\left.x I 0^{4}\right)$ and the top $\left(10-200 \mathrm{RLU} \times 10^{4}\right)$. Statistical significance was determined via two-way ANOVA and Tukey's post hoc test. (B and C) Cytotoxicity was measured by LDH assay and normalized to control for each donor. Data represent mean \pm SEM in 3-4 donors. Statistical significance was determined via one-way ANOVA followed by Tukey's post hoc $(* * p<0.01, * * * p<0.001)$. 


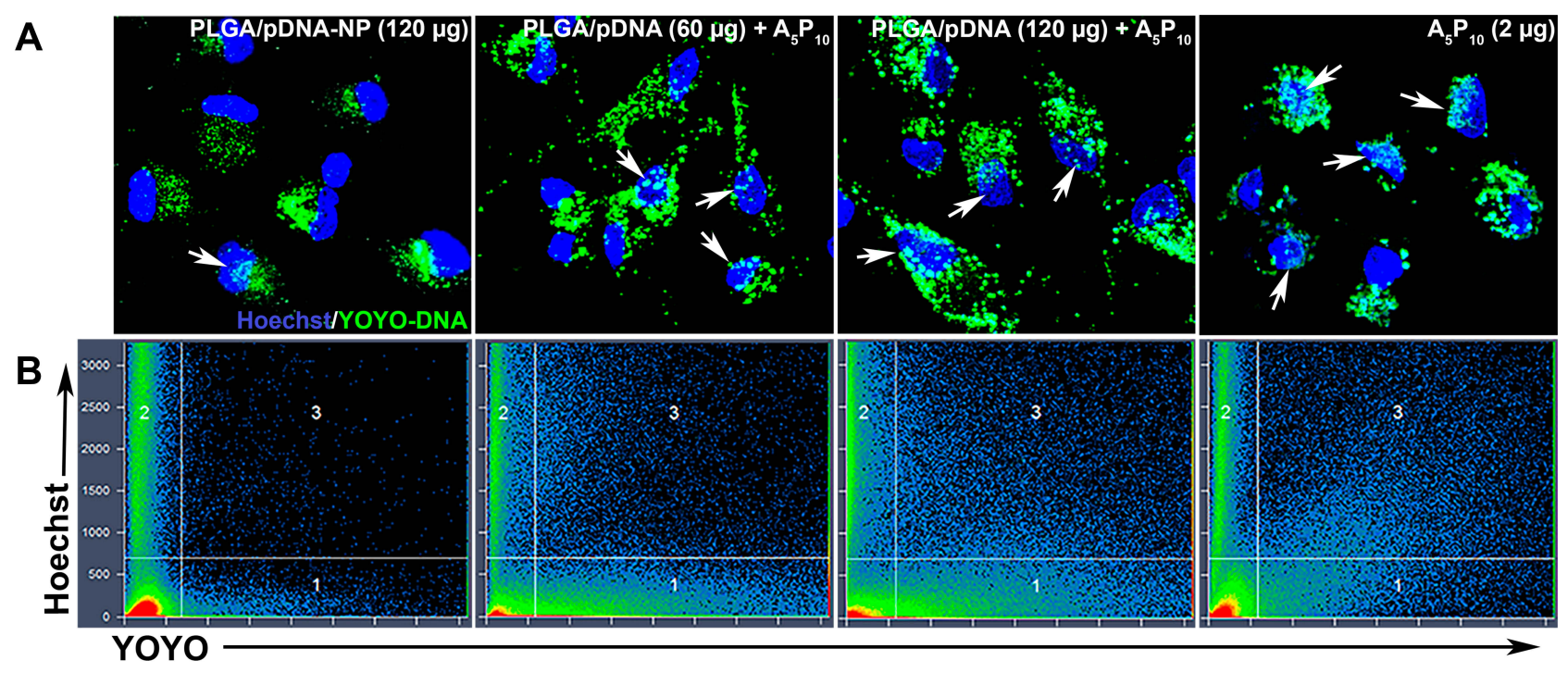

C

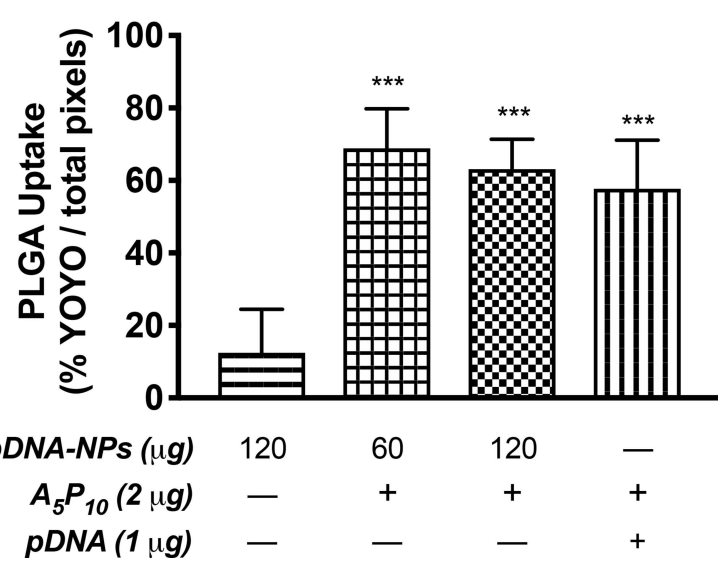

D

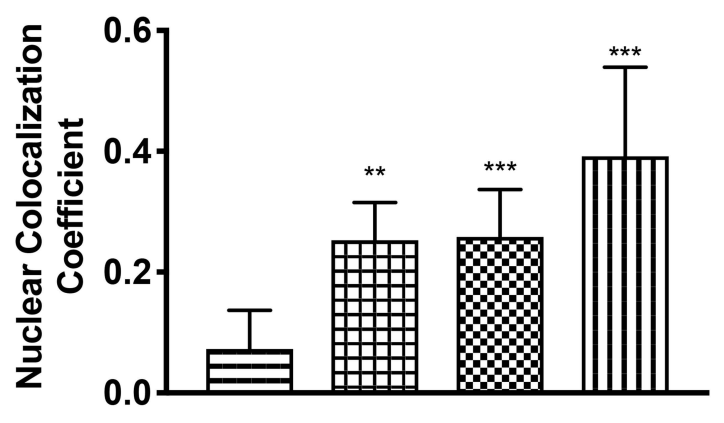

$\begin{array}{rcccc}\text { PLGA/PDNA-NPs }(\mu \mathrm{g}) & 120 & 60 & 120 & - \\ A_{5} P_{10}(2 \mu g) & - & + & + & + \\ \text { PDNA }(1 \mu g) & - & - & - & +\end{array}$

Figure $3 \mathrm{~A}_{5} \mathrm{P}_{10}$ increased PLGA/PDNA-NP-mediated uptake, intracellular trafficking, and nuclear delivery of pDNA. Primary human astrocytes were treated with PLGA/ PDNA-NPs $(60$ or $120 \mu \mathrm{g})$ alone or in combination with $2 \mu \mathrm{g} \mathrm{A}_{5} \mathrm{P}_{10}$ for 24 hours. $\mathrm{A}_{5} \mathrm{P}_{10}$ coupled with YOYO-pDNA was used as a positive control. Confocal images $(\mathbf{A})$ and colocalization analysis (B) were achieved using YOYO-pDNA and Hoechst nuclear dye. White arrows indicate YOYO and Hoechst colocalization. Scatter regions $I$ and 2 depict pixels for YOYO and Hoechst channels, respectively, and scatter region 3 determines the pixels colocalizing between the two channels. (C) Graphs report mean \pm SD for calculated total YOYO pixels divided by total pixels per image and (D) weighted colocalization coefficients in relation to total Hoechst pixels per image from a minimum of 8-10 images of triplicate wells per condition. Statistical significance was determined via one-way ANOVA followed by Tukey's post hoc test $(* * p<0.0 \mathrm{I}, * * * p<0.00 \mathrm{I})$.

a five-fold increase in the total intracellular YOYO-pDNA (Figure $3 \mathrm{C}, \mathrm{p}<0.001$ ) as well as nuclear localization (Figure $3 \mathrm{D}, \mathrm{p}<0.01)$. These findings demonstrate $\mathrm{A}_{5} \mathrm{P}_{10}$ enhanced PLGA/pDNA-NP-mediated uptake, trafficking, and nuclear delivery of pDNA to human astrocytes.

\section{Discussion}

Establishing an effective and biocompatible gene delivery system to astrocytes provides a promising prospect for medical interventions against debilitating neurological diseases and disorders. As PLGA is an FDA-approved polymer, PLGA/pDNA-NPs are favorable for clinical translation and have additional therapeutic advantages such as biodegradability and sustained release. ${ }^{6-8,11}$
However, our studies identified an unexpected limitation in PLGA/pDNA-NP-mediated gene delivery to primary human astrocytes despite success in other cell lines. ${ }^{9-11}$ By co-transfecting PLGA/pDNA-NPs with $\mathrm{A}_{5} \mathrm{P}_{10}$, we were able to significantly enhance gene expression in astrocytes by increasing NP uptake and pDNA nuclear localization while maintaining biocompatibility in both astrocytes and neurons. To our knowledge, our study is the first to investigate PLGA-PEI co-polymer gene delivery in primary human cells, as well as, to validate enhanced nuclear localization of pDNA as a mediating mechanism.

It is well established that a fundamental benefit of PLGA-NPs as a gene delivery system is sustained 
expression of the cargo for up to a week. ${ }^{6-8,11}$ For both PLGA/pDNA-NPs ${ }^{6-8,11}$ and $\mathrm{A}_{n} \mathrm{P}_{\mathrm{n}} / \mathrm{pDNA} \mathrm{NPs},{ }^{15}$ expression was measurable at 48 hours following exposure. Thus, this report focused on a 48-hour timepoint for gene expression assessment. However, our previous investigations characterizing PLGA/pDNA-NPs demonstrate that although most DNA release occurs in the first week, it has been quantified up to 15 days post-treatment. ${ }^{11}$ While the DNA release kinetics have not been evaluated for $A_{n} P_{n}$ /pDNA-NPs, we have reported detectable levels of gene expression through seven days. ${ }^{14,15}$ In this context, we expect the $\mathrm{PLGA} / \mathrm{pDNA}+\mathrm{A}_{5} \mathrm{P}_{10}$ to have similar gene expression kinetics in astrocytes and perhaps even improve upon the sustained expression achieved by either polymer alone.
Other NP studies have also explored the integration of PLGA-PEI constructs due to their complementary advantages and disadvantages. Consistent with our findings, these studies reported improved delivery and/or decreased cytotoxicity compared to PLGA-NPs or PEI alone. ${ }^{20-23}$ Our investigations provide further novelty with the application of arginine-modified PEI, which are not only significantly more biocompatible than unmodified PEI but also augment NP uptake and subsequent gene expression. ${ }^{14,15}$ Thus, the arginine additions of $\mathrm{A}_{5} \mathrm{P}_{10}$ could be a fundamental factor increasing cell entry of PLGA/pDNA-NPs during cotransfection. Moreover, PEI is known to increase chloride concentrations to induce endosomal swelling and promote endolysosomal escape; thus, as a PEI derivative, $\mathrm{A}_{5} \mathrm{P}_{10}$ may be enhancing intracellular pDNA survival. ${ }^{5,12,13}$

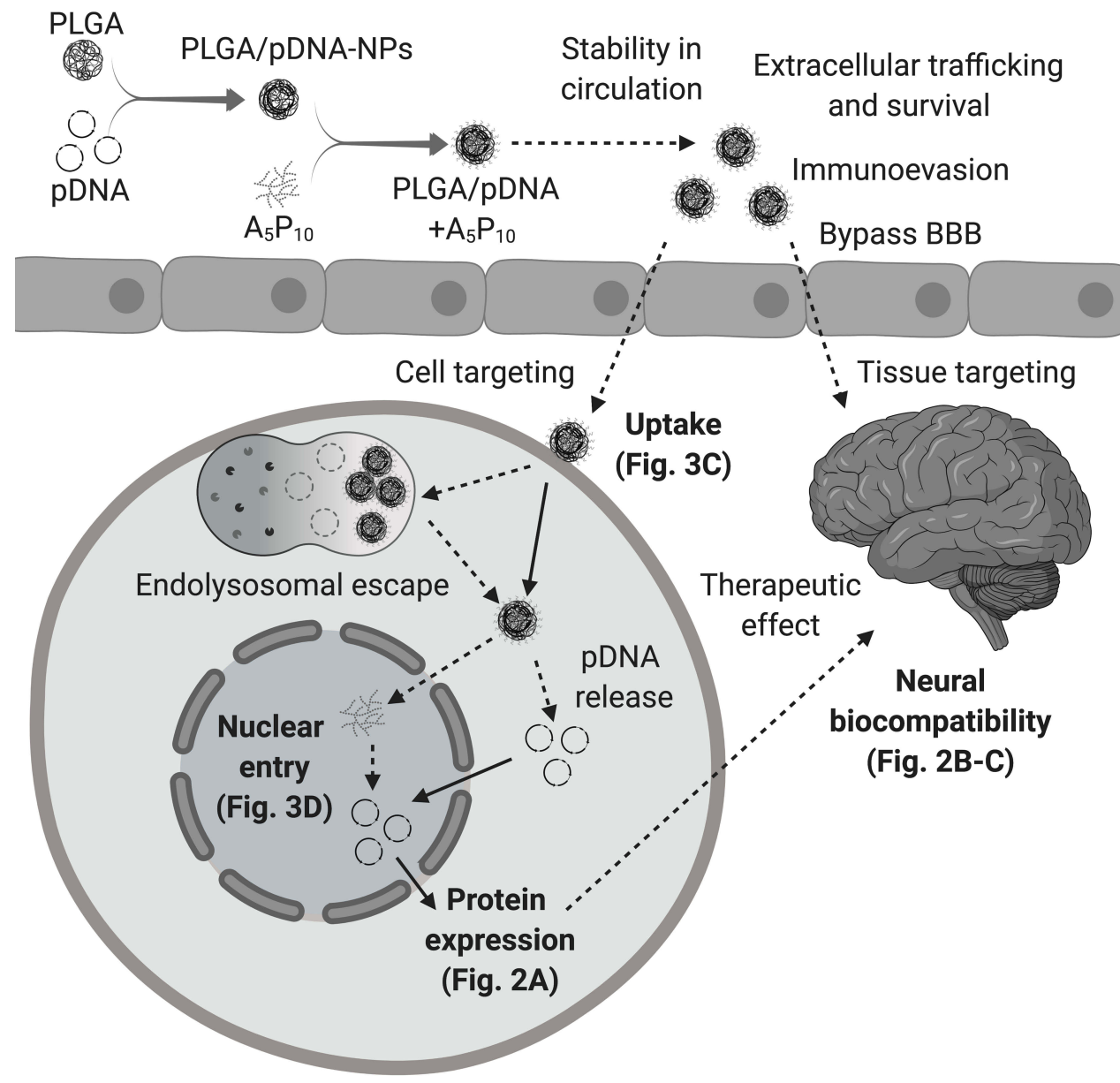

Figure 4 Obstacles for gene delivery to CNS. A successful gene delivery system to the CNS must achieve optimal trafficking and survival in the periphery including stability in circulation and evasion of the immune system. A critical dilemma for these constructs is bypassing the BBB in order to reach the tissue or cell of interest. Ensuring specificity is also of heightened importance for gene therapy applications. Once targeting is accomplished, delivery systems must overcome a variety of obstacles for intracellular trafficking including uptake, endolysosomal escape, nuclear entry, DNA release, and finally, expression of the therapeutic target. Moreover, systemic biocompatibility is an essential objective. Our report demonstrates (solid lines) PLGA/PDNA+ $A_{5} P_{10}$ was able to facilitate enhanced uptake and intracellular trafficking (Figure $3 \mathrm{C}$ ), nuclear entry of PDNA (Figure 3D), and exogenous gene expression (Figure 2A) while maintaining biocompatibility in astrocytes and neurons (Figure 2B and C). To translate this system for successful gene therapy, additional investigations are warranted to determine the potential of PLGA/pDNA+ $A_{5} P_{10}$ to overcome the remaining barriers and to further elucidate the intracellular mechanisms mediating PLGA/pDNA+ $A_{5} P_{10}$ optimization (dashed lines). (Created with BioRender.com). 
These characteristics of $\mathrm{A}_{5} \mathrm{P}_{10}$ rationalize the likely mechanisms driving the elevated pDNA presence in the cytoplasm of astrocytes. However, they do not explain how cotransfection promoted nuclear localization of pDNA, which remains a critical rate-limiting step and largely an enigmatic phenomenon for gene delivery systems. ${ }^{5,24-26}$ Others have proposed methods to overcome this limitation including the addition of nuclear localization sequences, characterized by positively charged basic amino acids (lysine, arginine, histidine), to the NP composition. ${ }^{5,24,25,27}$ In this context, the arginine residues on $\mathrm{A}_{5} \mathrm{P}_{10}$ may be promoting nuclear proximity or even assisting with nuclear entry. ${ }^{14}$ Moreover, the released pDNA is likely condensed by the positive charge of $\mathrm{A}_{5} \mathrm{P}_{10}$ in the cytoplasm prior to its transport to the nucleus. These findings provide a novel strategy to enhance nuclear entry of pDNA to optimize polymeric NP gene therapy systems.

\section{Conclusion}

A successful gene delivery system must overcome a series of obstacles which are described in Figure 4. Our findings (solid lines) identify an improved method that enhanced NP uptake, pDNA nuclear trafficking, and exogenous gene expression in astrocytes. Furthermore, PLGA- $\mathrm{A}_{5} \mathrm{P}_{10^{-}}$ mediated delivery was cytocompatible with primary human astrocytes and neurons, which supports safe in vivo translation of this NP delivery system. However, further studies are warranted to establish whether this system is capable of overcoming the remaining obstacles for astrocyte gene therapy (dotted lines), such as BBB permissibility, astrocyte specificity, sustainability, and in vivo biocompatibility. Several studies describe polymeric NP surface modifications that could help eliminate cytotoxicity and/or enhance BBB permissibility such as coating with silica, polyethylene glycol, or a ligand for BBB receptor targeting. ${ }^{6,23,28,29}$ Direct brain injection of PLGA-based NPs combined with $\mathrm{A}_{n} \mathrm{P}_{\mathrm{n}}$ is also an option. Furthermore, to accommodate astrocyte specificity, we recently reported the application of an astrocyte-specific protein promoter, glial fibrillary acid protein, to restrict expression of therapeutic genes. ${ }^{15}$ Moving forward, our findings offer an innovative avenue for astrocyte-targeted gene therapy and harbor the potential to optimize other therapeutic gene delivery systems.

\section{Abbreviations}

$\mathrm{A}_{\mathrm{n}} \mathrm{P}_{\mathrm{n}}$, arginine-modified polyethylenimine-based polymers; $\mathrm{BBB}$, blood-brain barrier; CNS, central nervous system; FDA, Federal Drug Administration; HEKs, human embryonic kidney 293 T cells; LDH, lactate dehydrogenase; NPs, nanoparticles; pDNA, plasmid DNA; PEI, polyethylenimine; PLGA, poly (lactide-co-glycolide); PLGA-NPs, poly (lactide-co-glycolide)-based nanoparticles; PLGA/ pDNA-NPs, poly (lactide-co-glycolide)-based nanoparticles encapsulating plasmid DNA; PLGA/pDNA $+\mathrm{A}_{5} \mathrm{P}_{10}$, poly (lactide-co-glycolide)-based nanoparticles encapsulating plasmid DNA treated in combination with $\mathrm{A}_{5} \mathrm{P}_{10}$; YOYO-pDNA, YOYO-labeled luciferase plasmid DNA.

\section{Acknowledgments}

The authors appreciate Satomi Stacy for providing consistent high-quality primary human tissue cultures and Venkata Viswanadh Edara, Naomi Swanta, and Dr. Rebecca Cunningham for critical reading of the manuscript. Special additional thanks to Ms. Stacy for technical and editing assistance.

\section{Author Contributions}

All authors contributed to the experimental design, acquisition of data, or analysis and interpretation of data. Nanoparticle and polymer formulations were developed and produced by VL, SV, and MS. Experiments were performed by both JP and CJ. Manuscript text was drafted by JP. All authors participated in editing or revising the article, gave final approval of the version to be published, and agreed to be accountable for all aspects of the work.

\section{Funding}

This study was funded by NINDS, R01 NS048837 to AG and KB. JP was additionally supported by NINDS T32 AG020494 Neurobiology of Aging Associate and Full Fellowships. We appreciate the assistance of the Laboratory of Developmental Biology for providing human brain tissues; supported by NIH 5R24 HD0008836 from the Eunice Kennedy Shriver National Institute of Child Health \& Human Development.

\section{Disclosure}

CJ's current affiliation is Department of Neurology and Neurotherapeutics at the University of Texas Southwestern Medical Center, Dallas, TX, USA. SV's current affiliation is Central Research Facility, Sri Ramachandra Institute of Higher Education and Research, Porur, Chennai, India. MS's current affiliation is the Department of Biomaterial Science and Technology, Sree Chitra Tirunal Institute for Medical Science and Technology, Trivandrum, Kerala, India. The authors declare that the research was conducted in the absence of any 
commercial or financial relationships that could be construed as a potential conflict of interest.

\section{References}

1. Sofroniew MV, Vinters HV. Astrocytes: biology and pathology. Acta Neuropathol. 2010;119(1):7-35. doi:10.1007/s00401-009-0619-8

2. Joshi CR, Labhasetwar V, Ghorpade A. Destination brain: the past, present, and future of therapeutic gene delivery. $J$ Neuroimmune Pharmacol. 2017;12(1):51-83. doi:10.1007/s11481-016-9724-3

3. Luten J, van Nostrum CF, De Smedt SC, Hennink WE. Biodegradable polymers as non-viral carriers for plasmid DNA delivery. J Control Release. 2008;126(2):97-110. doi:10.1016/j. jconrel.2007.10.028

4. Midoux P, Breuzard G, Gomez JP, Pichon C. Polymer-based gene delivery: a current review on the uptake and intracellular trafficking of polyplexes. Curr Gene Ther. 2008;8(5):335-352. doi:10.2174/ 156652308786071014

5. Jayant RD, Sosa D, Kaushik A, et al. Current status of non-viral gene therapy for CNS disorders. Expert Opin Drug Deliv. 2016;13 (10):1433-1445. doi:10.1080/17425247.2016.1188802

6. Danhier F, Ansorena E, Silva JM, Coco R, Le Breton A, Preat V. PLGAbased nanoparticles: an overview of biomedical applications. J Control Release. 2012;161(2):505-522. doi:10.1016/j.jconrel.2012.01.043

7. Patel T, Zhou J, Piepmeier JM, Saltzman WM. Polymeric nanoparticles for drug delivery to the central nervous system. Adv Drug Deliv Rev. 2012;64(7):701-705. doi:10.1016/j.addr.2011.12.006

8. Chen J, Guo Z, Tian H, Chen X. Production and clinical development of nanoparticles for gene delivery. Mol Ther Methods Clin Dev. 2016;3:16023. doi: $10.1038 / \mathrm{mtm} .2016 .23$

9. Prabha S, Sharma B, Labhasetwar V. Inhibition of tumor angiogenesis and growth by nanoparticle-mediated p 53 gene therapy in mice. Cancer Gene Ther. 2012;19(8):530-537. doi:10.1038/cgt.2012.26

10. Prabha S, Labhasetwar V. Nanoparticle-mediated wild-type p53 gene delivery results in sustained antiproliferative activity in breast cancer cells. Mol Pharm. 2004;1(3):211-219.

11. Prabha S, Labhasetwar V. Critical determinants in PLGA/PLA nanoparticle-mediated gene expression. Pharm Res. 2004;21 (2):354-364. doi:10.1023/B:PHAM.0000016250.56402.99

12. Sonawane ND, Szoka FC, Verkman AS. Chloride accumulation and swelling in endosomes enhances DNA transfer by polyamine-DNA polyplexes. J Biol Chem. 2003;278(45):44826-44831. doi:10.1074/ jbc.M308643200

13. Ramamoorth M, Narvekar A. Non viral vectors in gene therapy- an overview. J Clin Diagn Res. 2015;9(1):GE01-06. doi:10.7860/JCDR/ 2015/10443.5394

14. Morris VB, Labhasetwar V. Arginine-rich polyplexes for gene delivery to neuronal cells. Biomaterials. 2015;60:151-160. doi:10.1016/j. biomaterials.2015.04.052

15. Joshi CR, Raghavan V, Vijayaraghavalu S, et al. Reaching for the stars in the brain: polymer-mediated gene delivery to human astrocytes. Mol Ther Nucleic Acids. 2018;12:645-657. doi:10.1016/ j.omtn.2018.06.009

International Journal of Nanomedicine

\section{Publish your work in this journal}

The International Journal of Nanomedicine is an international, peerreviewed journal focusing on the application of nanotechnology in diagnostics, therapeutics, and drug delivery systems throughout the biomedical field. This journal is indexed on PubMed Central, MedLine, CAS, SciSearch ${ }^{\mathbb{R}}$, Current Contents ${ }^{\mathbb{B}} /$ Clinical Medicine,
16. Lu S, Morris VB, Labhasetwar V. Effectiveness of siRNA delivery via arginine-rich PEI-based polyplex in metastatic and doxorubicin resistant breast cancer cells. J Pharmacol Exp Ther. 2019;370 (3):902-910. doi:10.1124/jpet.119.256909

17. Gardner J, Borgmann K, Deshpande MS, et al. Potential mechanisms for astrocyte-TIMP-1 downregulation in chronic inflammatory diseases. J Neurosci Res. 2006;83(7):1281-1292. doi:10.1002/ jnr.20823

18. Reddy MK, Wu L, Kou W, Ghorpade A, Labhasetwar V. Superoxide dismutase-loaded PLGA nanoparticles protect cultured human neurons under oxidative stress. Appl Biochem Biotechnol. 2008;151(2-3):565-577. doi:10.1007/s12010-008-8232-1

19. Singhal A, Morris VB, Labhasetwar V, Ghorpade A. Nanoparticlemediated catalase delivery protects human neurons from oxidative stress. Cell Death Dis. 2013;4:e903. doi:10.1038/cddis.2013.362

20. Zhang XQ, Intra J, Salem AK. Comparative study of poly (lactic-coglycolic acid)-poly ethyleneimine-plasmid DNA microparticles prepared using double emulsion methods. J Microencapsul. 2008;25 (1):1-12. doi:10.1080/02652040701659347

21. Shau MD, Shih MF, Lin CC, et al. A one-step process in preparation of cationic nanoparticles with poly(lactide-co-glycolide)-containing polyethylenimine gives efficient gene delivery. Eur J Pharm Sci. 2012;46(5):522-529. doi:10.1016/j.ejps.2012.04.006

22. Lu JM, Liang Z, Wang X, Gu J, Yao Q, Chen C. New polymer of lactic-co-glycolic acid-modified polyethylenimine for nucleic acid delivery. Nanomedicine. 2016;11(15):1971-1991. doi:10.2217/nnm2016-0128

23. Kolte A, Patil S, Lesimple P, Hanrahan JW, Misra A. PEGylated composite nanoparticles of PLGA and polyethylenimine for safe and efficient delivery of pDNA to lungs. Int J Pharm. 2017;524(1-2):382-396. doi:10.1016/j.ijpharm.2017.03.094

24. Panyam J, Labhasetwar V. Biodegradable nanoparticles for drug and gene delivery to cells and tissue. Adv Drug Deliv Rev. 2003;55 (3):329-347. doi:10.1016/S0169-409X(02)00228-4

25. Lungwitz U, Breunig M, Blunk T, Gopferich A. Polyethyleniminebased non-viral gene delivery systems. Eur J Pharm Biopharm. 2005;60(2):247-266. doi:10.1016/j.ejpb.2004.11.011

26. Yin H, Kanasty RL, Eltoukhy AA, Vegas AJ, Dorkin JR, Anderson DG. Non-viral vectors for gene-based therapy. Nat Rev Genet. 2014;15(8):541-555. doi:10.1038/nrg3763

27. Lange A, Mills RE, Lange CJ, Stewart M, Devine SE, Corbett AH. Classical nuclear localization signals: definition, function, and interaction with importin alpha. J Biol Chem. 2007;282(8):5101-5105. doi:10.1074/jbc.R600026200

28. Morris VB, Sharma CP. Enhanced in-vitro transfection and biocompatibility of L-arginine modified oligo (-alkylaminosiloxanes)-graftpolyethylenimine. Biomaterials. 2010;31(33):8759-8769. doi:10.1016/j.biomaterials.2010.07.073

29. Mastorakos P, Zhang C, Berry S, et al. Highly PEGylated DNA nanoparticles provide uniform and widespread gene transfer in the brain. Adv Healthc Mater. 2015;4(7):1023-1033. doi:10.1002/ adhm. 201400800 